\title{
Utilización del modelo Brody para describir el crecimiento de dos grupos raciales de ovinos en Córdoba, Colombia ${ }^{1}$
}

\section{ARTÍCULO DE INVESTIGACIÓN}

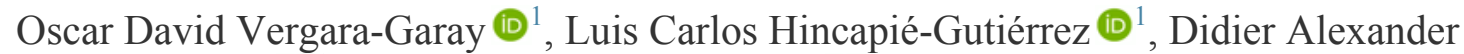
Vallejo-Romero $\mathbb{C}^{1}$, Juan Carlos Simanca-Sotelo $\mathbb{C}^{1}$, Moris de Jesús Bustamante-Yánez (1) ${ }^{1}$

1 Grupo de Investigación en Producción Animal Tropical, Facultad de Medicina Veterinaria y Zootecnia, Universidad de Córdoba, Colombia.

overgara@correo.unicordoba.edu.co

Recibido: 2 de Octubre de 2016 y aprobado: 10 de Febrero de 2017, Actualizado: 12 de Junio de 2017

DOI: 10.17151/vetzo.2017.11.1.1

RESUMEN: Las curvas del crecimiento reflejan la relación entre la edad del animal y el desarrollo propio del individuo para crecer y madurarse corporalmente en el ambiente en el cual se desarrolla. El objetivo de este trabajo fue evaluar el crecimiento de ovinos Criollos y F1 Santa Inés por Criollo, utilizando el modelo no lineal de Brody en una granja del municipio de Montería (Córdoba, Colombia). Se utilizaron pesajes de 40 animales, de los cuales 15 eran criollos y 25 Santa Inés por criollo. Los animales fueron pesados desde el nacimiento hasta los seis meses de edad. Se realizó un ajuste del modelo mediante el procedimiento NLIN de Statistical Analysis Software (SAS), para la estimación de los parámetros del modelo. Además, se estimó el porcentaje de madurez a los cuatro y seis meses de edad y edad al 75 y $95 \%$ de madurez. Se realizaron análisis de varianzas para evaluar los efectos grupo genético y sexo sobre los parámetros del modelo y sobre los porcentajes y edad a la madurez. Los parámetros estimados fueron $42,0 \pm 10,6,0,932 \pm 0,0444$ y $0,00528 \pm 0,00242$ para $\beta_{0}, \beta_{1}$ y $\beta_{2}$, respectivamente. El porcentaje de madurez a los cuatro y seis meses fue de 48,3 y $60,5 \%$ y la edad al 75 y $95 \%$ de madurez fue de 305 y 675 días, respectivamente. De acuerdo con los resultados, el sexo tuvo efecto significativo $(\mathrm{P} \leq 0,05)$ sobre $\beta_{2}$ y los porcentajes de madurez; mientras que, el efecto grupo genético fue significativo $(\mathrm{P} \leq 0,05)$ solo para $\beta_{0}$.

Palabras clave: edad a la madurez, modelo no lineal, ovinos criollos, porcentaje de madurez, Santa Inés

\section{Using the model Brody to describe the growth of two racial groups of sheep in Cordoba, Colombia}

ABSTRACT: Growth curves reflect the relationship between the age of the animal and individual development to grow and mature corporeal in the environment in which it is developed. The objective of this study was to evaluate the growth of Creole sheep and F1 Santa Inés by Creole using the nonlinear model of Brody on a farm in the municipality of Monteria (Córdoba, Colombia). Weights of 40 animals 
were used, of which 15 were Creoles and 25 Santa Ines by Creole. The animals were weighed from birth until six months of age. A model adjustment was made using the NLIN procedure of Statistical Analysis Software (SAS), to estimate the model parameters. Moreover, the percentage of maturity was estimated at four and six months of age and age at 75 and $95 \%$ of maturity. Analysis of variance were performed to evaluate the genetic group and sex effects on the model parameters and percentages and age at maturity. The estimated parameters were $42.0 \pm 10.7,0.932 \pm 0.0444$ and $0.00528 \pm 0.00242$ for $\beta_{0}, \beta_{1}$ and $\beta_{2}$, respectively. The percentage of maturity at four and six months was 48.3 and $60.5 \%$ and age at 75 and $95 \%$ maturity was 305 and 675 days, respectively. According to the results, sex had a significant effect $(\mathrm{P} \leq 0.05)$ on $\beta_{2}$ and percentages of maturity; while genetic group effect was significant $(\mathrm{P} \leq 0.05)$ only for $\beta_{0}$.

Key words: growth curve, nonlinear model, Santa Ines, Sheep creole

\section{Introducción}

Los ovinos han constituido una especie que provee variadas satisfacciones a la humanidad; lana, carne, piel o leche son algunos de los elementos explotados por el hombre (López et al., 2000), siendo la producción de carne la principal actividad desarrollada en el país. Vale resaltar que esta especie viene presentando gran expansión y actualmente según el inventario del Instituto Colombiano Agropecuario, la población ovina para el año 2015 era de 1.318.241 animales en el territorio nacional (ICA, 2015).

La escasa definición de políticas gubernamentales, así como la poca investigación en el sector ovino, asociado a la resistencia de la gran mayoría de productores a modificar y optimizar sus procesos y procedimientos tradicionales, no han propiciado desarrollos tecnológicos y empresariales para los productores ovinos, que en su mayoría manejan sistemas de producción extensivos, sin definición de objetivos, sin control productivo, escasa o nula información, problemas de endogamia, baja productividad $\mathrm{y}$ desconocimiento de la calidad de los productos obtenidos (Vivas, 2013), provocando problemas como la pasividad productiva ovina, por dejar la selección y mejora genética para esta especie asociada al esfuerzo de pocos investigadores y ganaderos, presentándose un paulatino crecimiento del sector.

Otro inconveniente que presentan los productores de ovinos es no contar con parámetros de crecimiento (peso al nacimiento, peso al destete y peso al sacrificio), que permitan tomar decisiones estratégicas, pudiendo determinar las edades de sacrificio que permitan obtener el máximo beneficio económico, el peso al alcanzar la madurez sexual, la tasa de crecimiento con respecto a la tasa de maduración sexual, y esto solo puede ser comparado y evaluado teniendo en cuenta la información zootécnica de registros asociados a investigaciones ovinas (Vivas, 2013). Esto hace de vital importancia realizar estudios para profundizar en el conocimiento sobre la tasa reproductiva, la velocidad del crecimiento y la calidad de la canal de los ovinos desde el nacimiento hasta su fase adulta. 
Por otro lado, dentro de las medidas de crecimiento corporal animal, una de las más comunes, que no altera el organismo bajo análisis y que puede ser medida a bajo costo, es el peso en determinadas edades, con el que podemos inferir el crecimiento animal por medio de modelos matemáticos no-lineales, capaces de predecir el desempeño de la evolución del peso vivo en la descripción de la curva de crecimiento; dichas funciones permiten realizar evaluaciones sobre el nivel de producción en las empresas ovinas, pudiendo clasificar de forma sencilla la productividad de una raza específica para una zona determinada (Agudelo et al., 2008).

Las curvas del crecimiento reflejan la relación entre la edad del animal y el impulso propio del individuo para crecer hasta obtener su peso adulto y conformación propia de la especie, en el ambiente en el cual estos impulsos están expresados (Fitzhugh, 1976). El poder predecir el crecimiento animal mediante el uso de modelos matemáticos y establecer estrategias como planes de alimentación y manejo en cada etapa productiva, al igual que en la reproducción en los diferentes sistemas de producción, es indicado para procurar la obtención de rendimientos ideales, por lo que el conocimiento de las curvas de crecimiento animal es importante en el monitoreo de un rebaño productivo, ya que expresan gran cantidad de información inherente al animal durante toda su vida (Pineda et al., 2013). Varios son los estudios que han utilizado el modelo Brody para describir el crecimiento de poblaciones ovinas de diferentes razas y cruces (Andrade et al., 2011; Bahreini et al., 2014; Teixeira et al., 2016); por lo que, el objetivo principal de este estudio fue determinar la curva de crecimiento en ovinos (Ovis aries) criollos de pelo colombiano y F1 de ovinos Santa Inés x criollo en condiciones de pastoreo rotacional en el departamento de Córdoba mediante el modelo de regresión no lineal de Brody.

\section{Materiales y Métodos}

\section{Localización}

Los datos obtenidos para la realización del estudio se tomaron en ovinos de un sistema de producción ubicado en el municipio de Montería, Córdoba (Colombia). Este municipio se encuentra ubicado a $8^{\circ} 45^{\prime}$ latitud Norte y a $75^{\circ} 53^{\prime}$ longitud Oeste, con una altitud aproximada de $20 \mathrm{msnm}$, una precipitación anual de $1156 \mathrm{~mm}$, una temperatura media anual de $28^{\circ} \mathrm{C}$ y humedad relativa de $85 \%$.

\section{Animales y datos}

Para este estudio se utilizaron 15 ovinos criollos de pelo colombiano (9 machos y 6 hembras) y 25 F1 de Santa Inés x ovinos criollos de pelo colombiano (11 machos y 14 hembras), provenientes de partos sencillos. Para la toma de datos en campo, los animales fueron pesados al nacer y después cada 15 días de edad, hasta los seis meses de edad (el destete se realizó a los 90 días). Para realizar los pesajes se utilizó una báscula digital JAZ-DINA L150 con capacidad para $150 \mathrm{~kg}$. Los animales fueron manejados bajo condiciones de pastoreo rotacional en praderas de pasturas de Bothriochloa pertusa yBraquiaria brizanta, con un periodo de ocupación de 7 días y un periodo de descanso de 24 días, con disponibilidad de agua y sal mineral a voluntad. Además, se suplementaba con ensilaje de maíz. 


\section{Análisis de datos}

Los datos se tabularon en una base de datos de Microsoft Excel y para realizar el ajuste de los datos se utilizó el modelo no lineal propuesto por Brody (1946), mediante el procedimiento NLIN del programa estadístico SAS (2007). El modelo no lineal que se utilizó para describir el crecimiento fue el siguiente:

$$
y_{i}=\beta_{0}\left(1-\beta_{1} e^{\beta_{2} t}\right)
$$

Donde:

yi representa el i-ésimo peso del animal en el i-ésimo tiempo ti;

$\beta_{0}$, es el peso asintótico cuando tiende a infinito;

$\beta_{1}$ es un parámetro de ajuste cuando $\mathrm{y} \neq 0$ ó $\mathrm{t} \neq 0$;

$\beta_{2}$ es un índice de madurez expresado como una proporción de porcentaje del máximo crecimiento con respecto al peso adulto del animal. Hay que considerar que se validó el supuesto de normalidad e independencia de los errores

Además, se calculó el porcentaje de madurez a los cuatro (\%M4) y seis meses (\%M6) y edad al 75\% (EM75) y 95\% (EM95) de madurez para la población ovina estudiada, de acuerdo con las siguientes fórmulas:

$$
\% M=\left(1-\beta_{1} e^{\beta_{2} t}\right)
$$

Dónde:

$\% \mathrm{M}=$ porcentaje de madurez observado a 4 ó 6 meses;

$\mathrm{t}=$ tiempo para alcanzar un porcentaje de madurez (4 ó 6 meses).

$$
\left.E M=-\log \left((1-\% M) / \beta_{1}\right) /-\beta_{2}\right)
$$

Dónde:

$\mathrm{EM}=$ edad al alcanzar el 75 ó 95\% de madurez;

$\% \mathrm{M}=$ porcentaje de madurez esperado (75 ó 95\%).

Para determinar el efecto sexo y grupo genético sobre los parámetros del modelo y las edades y porcentajes de madurez, se utilizó un análisis de varianza, el cual se realizó mediante el procedimiento GLM de SAS (2007), mediante el siguiente modelo:

$$
y_{i j}=\mu+G_{i}+S_{j}+\varepsilon_{i j}
$$

Donde:

$\mathrm{y}_{\mathrm{ij}}$ : parámetros del modelo o las edades de madurez o porcentajes de madurez;

$\mu$ : media general a todas las observaciones;

$\mathrm{G}_{\mathrm{i}}$ : efecto del i-ésimo grupo genético;

$\mathrm{S}_{\mathrm{j}}$ : efecto del j-ésimo sexo;

$\varepsilon_{\mathrm{ij}}$ : residual 


\section{Resultados y Discusión}

En la Tabla 1 se presentan los promedios y desviaciones estándar para los parámetros, edades y porcentajes de madurez de acuerdo al modelo Brody. La Figura 1 muestra la tendencia del crecimiento de los animales de las poblaciones estudiadas de acuerdo al modelo mencionado; en dicha figura, se hace una proyección del peso hasta los 720 días de edad, de acuerdo con los parámetros estimados del modelo. El coeficiente de determinación del modelo fue de 99,15.

Tabla 1. Promedios y desviaciones estándar de los estimados de los parámetros, de edades y porcentajes de madurez de acuerdo al modelo Brody.

\begin{tabular}{ccc}
\hline Variable & Media & Desviación estándar \\
\hline$\beta_{0}$ & 42 & 10,6 \\
$\beta_{1}$ & 0,932 & 0,0444 \\
$\beta_{2}$ & 0,00528 & 0,00242 \\
$\%$ M4 & 48,2 & 14,7 \\
$\%$ M6 & 60,5 & 15,4 \\
EM75(dias) & 305 & 140 \\
EM95 (días) & 675 & 302 \\
\hline
\end{tabular}

En el presente estudio, el valor de $\beta 0$ se encuentra por encima a los reportados por Rocha et al. (2006) en Ovinos Santa Inés (27,4), Andrade et al. (2011) en ovinos de la raza Morada Nova (29) y Costa et al. (2012) en tres poblaciones de ovinos Santa Inés $(30,1,31,3$ y 14,4). Valores superiores al presente estudio han sido reportados por Santos (2012) en diferentes grupos genéticos (45,2), Contini (2015) en ovinos de la raza Morada Nova (51,2), Costa et al. (2009), Acioli (2010) y Teixeira et al. (2016) en ovinos de la raza Santa Inés (49,3 $\pm 0,3,130,1$ y 48,9, respectivamente).

El valor de $\beta_{1}$ del presente estudio se encuentra por encima de los valores reportados por Rocha et al. (2006), Andrade et al. (2011), Costa et al. (2012), Santos (2012), Contini (2015) y Teixeira et al. (2016), quienes encontraron valores que oscilaron entre 0,887 y 0,924, pero se encuentra por debajo a los valores reportados por Acioli (2010) y Costa et al. (2009), que obtuvieron valores de 0,945 y $0,99 \pm 0,003$, respectivamente. 


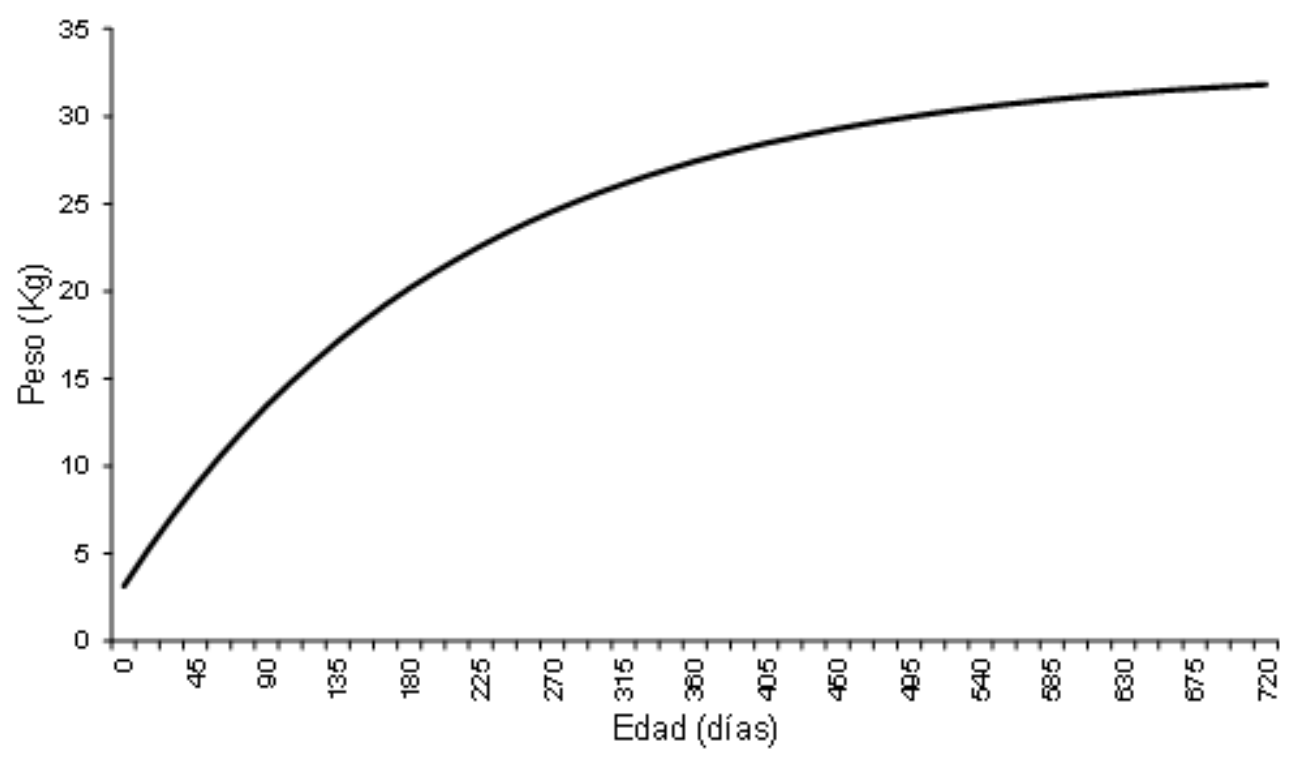

Figura 1. Comportamiento del peso en función de la edad en las poblaciones de ovinos estudiadas, de acuerdo con los parámetros estimados del modelo Brody.

El valor de $\beta_{2}$ del presente estudio se encuentra por encima de los valores reportados por Acioli (2010), Santos (2012) y Contini (2015) y de uno de los sistemas de producción estudiados por Costa et al. (2012), que obtuvieron valores que oscilaron entre 0,001 y 0,0041, respectivamente. Dato similar fue encontrado por Costa et al. (2009), quienes reportaron en su estudio $0,005 \pm 0,0007$, pero fue inferior a los valores reportados por Rocha et al. (2006), Andrade et al. (2011), Teixeira et al. (2016), y a dos de los sistemas de producción estudiados por Costa et al. (2012), quienes encontraron valores que oscilaron entre 0,006 y 0,0094 .

Respecto al porcentaje de madurez a los 4 meses, Llorente \& Ramos (2014) reportaron un porcentaje similar a este estudio $(46,5 \pm 4,6)$. Sin embargo, las edades al 75 y $95 \%$ de madurez fueron inferiores $(274 \pm 84$ y $596 \pm 201$ d). Las discrepancias encontradas en el presente estudio y la literatura citada, tanto para los parámetros del modelo como para los estimados de edad a la madurez y porcentaje de madurez, pueden atribuirse a las diferentes constituciones genéticas de los ovinos evaluados, y a las diferentes condiciones climatológicas e influencia nutricional proporcionadas en cada uno de los estudios referenciados en este trabajo, que afectan el desarrollo corporal de los animales.

Por otro lado, cabe resaltar que todos los estimados, tanto de los parámetros del modelo como de edades y porcentajes de madurez presentaron desviaciones estándar altas, quizás a las variaciones en peso que presentaron los animales a las diferentes edades entre los grupos genéticos evaluados y entre sexo. Además, pudo influir el número de partos de la madre, información que no se consideró en el modelo, ya que no se disponía de ella.

En la Tabla 2 se presentan los promedios de acuerdo con el efecto sexo y grupo genético para los parámetros estimados del modelo Brody y las edades y porcentajes de madurez. Se encontró diferencia significativa $(\mathrm{P} \leq 0,05)$ para el parámetro $\beta 2$ del modelo Brody y para el porcentaje de madurez a los 4 y 6 meses para el efecto de sexo. El $\beta_{2}$ estimado 
fue superior en machos (Tabla 2), por lo que se considera que los machos son animales más precoces que las hembras, debido a que presentan el $\beta_{2}$ más alto en relación a las hembras.

Tabla 2. Promedios de acuerdo al sexo y grupo genético en las poblaciones estudiadas seguin el modelo Brody.

\begin{tabular}{|c|c|c|c|c|c|c|c|c|}
\hline Efecto & & $\beta_{0}$ & $\beta_{1}$ & $\beta_{2}$ & $\% \mathrm{M} 4$ & \%М6 & EM75(días) & EM95(días) \\
\hline \multirow{2}{*}{ Sexo } & Macho & 38,7 & 0,921 & $0,00622^{\mathrm{a}}$ & $53,6^{\mathrm{a}}$ & $65,9^{\mathrm{a}}$ & 265 & 589 \\
\hline & Hembra & 43,5 & 0,938 & $0,00460^{b}$ & $44,3^{b}$ & $56,4^{b}$ & 340 & 751 \\
\hline \multirow{2}{*}{$\begin{array}{l}\text { Grupo } \\
\text { genético }\end{array}$} & Criollo & $37,3^{\mathrm{a}}$ & 0,919 & 0,00592 & 51,9 & 63,8 & 292 & 648 \\
\hline & C X SI & $44,8^{b}$ & 0,940 & 0,00490 & 46,1 & 58,5 & 313 & 691 \\
\hline
\end{tabular}

En el presente estudio, para las hembras el valor de $\beta_{0}(43,5)$ fue inferior al reportado por Costa et al. (2009) y Teixeira et al. (2016), los cuales fueron de 49,3 y 45,1, respectivamente, siendo el valor de $\beta_{1}$ tanto para el actual estudio como para el realizado por Teixeira et al. (2016) de 0,921, pero inferior al reportado por Costa et al. (2009), el cual fue de 0,99. En cuanto a $\beta_{2}$, el valor del estudio actual fue inferior $(0,0046)$ al reportado por Costa et al. (2009) y Teixeira et al. (2016), los cuales fueron de 0,0067 y 0,005 . Para los machos, los valores reportados para $\beta_{0}$ y $\beta_{1}$ en el presente estudio fueron inferiores (Tabla 2) a los reportados por Teixeira et al. (2016) que fueron 53 y 0,928 , respectivamente. En cuanto a $\beta_{2}$, el valor del presente estudio fue superior $(0,00622)$ al reportado por Teixeira et al. (2016), el cual fue de 0,0059.

En cuanto al porcentaje de madurez, a los 4 y 6 meses los machos presentaron mayores valores que las hembras (Tabla 2), por lo que los machos presentaron un mayor rendimiento de crecimiento y desarrollo en su conjunto corporal que las hembras, lo que se explica por un mayor valor de $\beta_{2}$. Comparativamente los machos crecieron más rápido que las hembras (Figura 2), debido precisamente a la mayor potencia de los andrógenos con respecto a los estrógenos sobre la estimulación del crecimiento (Bavera et al. 2005), lo que se puede notar hasta los 180 días, el cual fue el periodo en el que se realizaron los pesajes. Sin embargo, para una mejor visualización del momento en que la curva se hace asintótica, esta se realizó hasta los 720 días. 


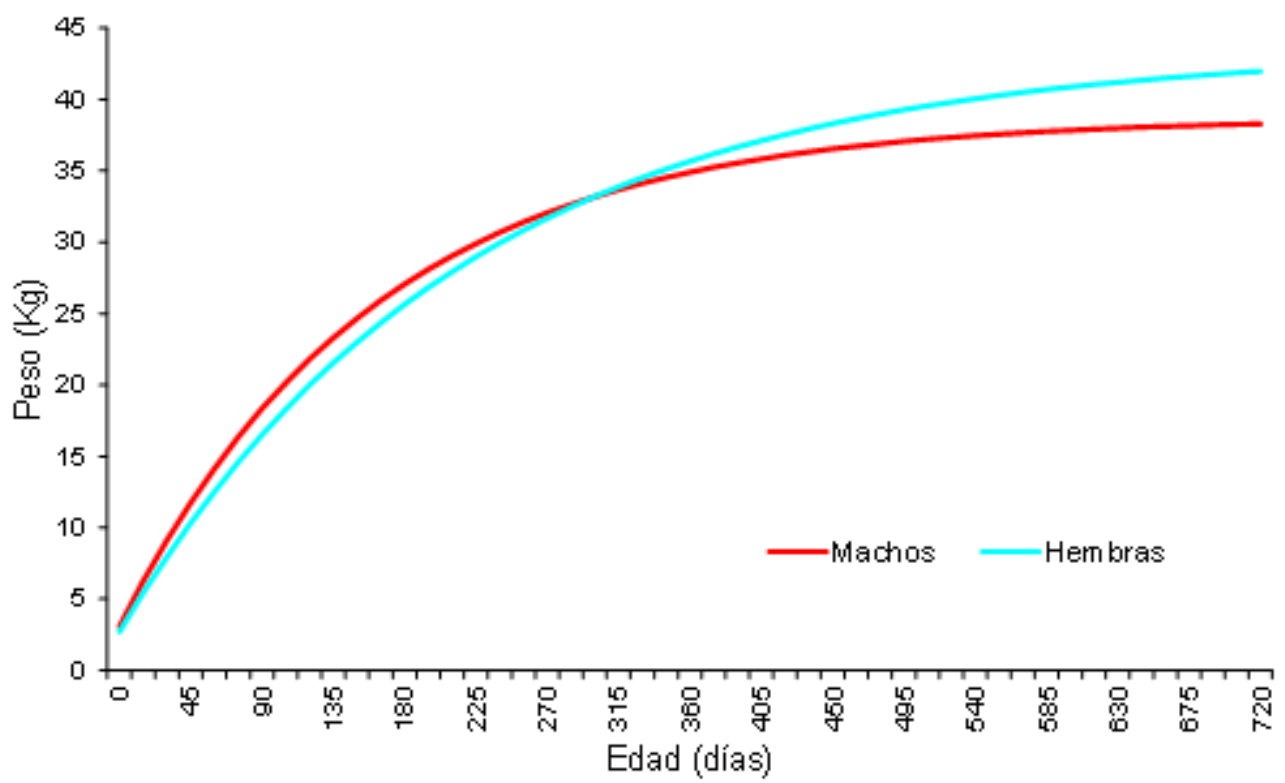

Figura 2. Curva de crecimiento por sexo, de acuerdo al modelo Brody en los ovinos estudiados.

Respecto al efecto del grupo genético, solo se encontró diferencia significativa $(\mathrm{P} \leq 0,05)$ para el parámetro $\beta_{0}$, por lo que se puede inferir de acuerdo con los resultados, que los animales F1 tuvieron un mayor peso adulto (Figura 3), quizás por el efecto de la heterosis.

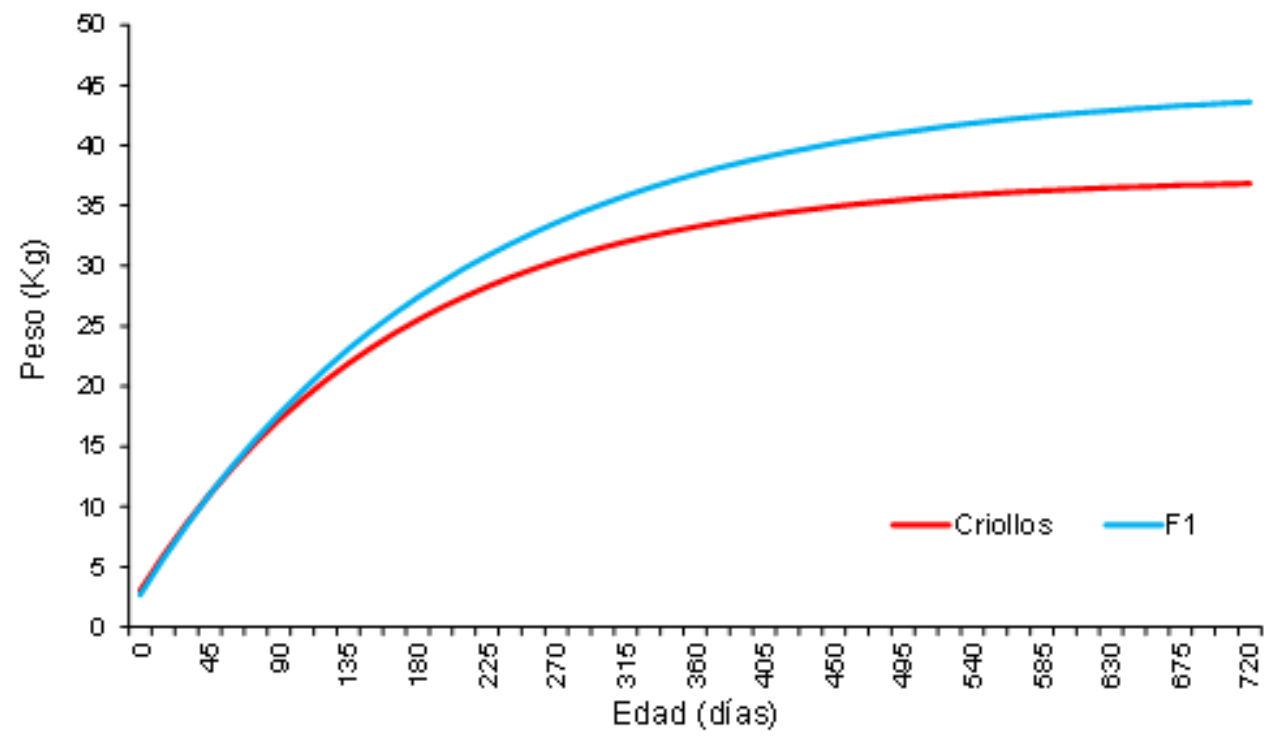

Figura 3. Curva de crecimiento por grupo genético de acuerdo al modelo Brody en los ovinos estudiados.

Por último, se puede afirmar que de acuerdo con la tasa de crecimiento estimada en los ovinos criollos, se hace necesaria la implementación de programas de mejoramiento 
genético para aumentar su peso adulto en busca de una mayor rentabilidad de los sistemas de producción.

\section{Conclusiones}

De acuerdo con los resultados, en las poblaciones ovinas estudiadas, los machos fueron más precoces que las hembras, explicado esto por el dimorfismo sexual. Además, se observó que los animales criollos alcanzan un menor peso adulto respecto a los F1 Santa Inés x criollo, debido a los efectos de la heterosis en los animales cruzados. Por otro lado, se debe seguir realizando estudios acerca de las características productivas que presenta el ovino criollo de pelo colombiano, para demostrar el potencial genético que estos animales poseen, y se sugiere para futuros estudios aumentar el número de animales a evaluar y el número de sistemas de producción.

\section{Agradecimientos}

Los autores agradecen al propietario y personal administrativo del sistema de producción, por facilitar la información para el desarrollo de esta investigación.

\section{Referencias bibliográficas}

- Acioli, L.A. Curva de crescimento em ovinos da raça Santa Inês. Alagoas, Brasil: Universidad Federal de Alagoas, 2010.47 p. Tese (Mestrado em Zootecnia). Disponible en: http://www.ufal.edu.br/unidadeacademica/ceca/posgraduacao/zootecnia/dissertacoes/laiza-sofia-acioli-da-silva. Accesado en: 10/07/2016.

- Agudelo, D.; Cerón, M.; Restrepo, L. Modelación de las funciones de crecimiento aplicadas a la producción animal. Revista Colombiana de Ciencias Pecuarias, v.21, n.1, p.39-58, 2008. Disponible en: http://rccp.udea.edu.co/index.php/ojs/article/viewFile/330/327. Accesado en: 10/07/2016.

- Andrade, L.; Souza, P.; Mendes, C. et al. Curvas de crescimento em ovinos da raça morada nova criados no estado da Bahia. Revista Brasileira de Zootecnia, v.40, n.8, 
p.1700-1705, 2011. Disponible en: http://www.scielo.br/pdf/rbz/v40n8/11.pdf. Accesado en: 10/07/2016.

- Bahreini, M.R.; Aslaminejad, A.A.; Sharifi, A.R. et al. Comparison of mathematical models for describing the growth of Baluchi sheep. Journal of Agricultural Science and Technology, v.14, p.57-68, 2014. Disponible en: http://jast.modares.ac.ir/article_10300_6dbbd6beee43147266f177a2af186e32.pdf. Accesado en: 14/07/2016.

- Bavera, G.; Bocco, O.; Beguet, H. et al. Crecimiento, desarrollo y precocidad. Sitio Argentino de Producción Animal Cursos de Producción Bovina de Carne, FAV UNRC. 2005. Disponible en: http://www.produccionanimal.com.ar/informacion_tecnica/exterior/05crecimiento_desarrollo_y_precocidad.pdf. Accesado en: 07/07/2016.

- Brody, S. Bioenergetics and growth. American Journal of Physical Anthropology, v.4, n.1, p.116-117, 1946

- Contini, E. Modelos não lineares no ajuste do crescimento de ovinos da raça Morada Nova. Nova Odessa, Brasil: Instituto de Zootecnia, 2015. 51p. Tese (Mestrado em produção animal sustentável). Disponible en: http://www.iz.sp.gov.br/pdfs/1438971752.pdf. Accesado en: 10/07/2016.

- Costa, V.; Quirino, C.R.; Dias, R.L. et al. Aplicação de modelos não lineares no ajuste de curvas de crescimento em fêmeas ovinas (Ovis aries) da raça Santa Inês criadas na região Norte Fluminense/RJ. Boletim de Indústria Animal, v.66, n.2, p. 115-120, 2009. Disponible en: http://revistas.bvs-vet.org.br/bia/article/view/24264/25140. Accesado en: 10/07/2016.

- Costa, M.C.; Villarroel, A.B.; Pereira, E.S. et al. Curva de crescimento de cordeiros oriundos de três sistemas de produção na Região Nordeste do Brasil. Ciências Agrárias, v.33, n.5, p. 2011-2018, 2012. Disponible en: http://www.uel.br/revistas/uel/index.php/semagrarias/article/viewFile/9655/11580.Acce sado en: 10/07/2016.

- Fitzhugh, H. Analysis of growth curves and strategies for altering their shape. Journal of Animal Science, v.42, n.4, p.1036-1051, 1976.

- Instituto Colombiano Agropecuario. Censo ovino de Colombia, 2015. Disponible en: www.ica.gov.co/getdoc/8232c0e5-be97-42bd-b07b-9cdbfb07fcac/Censos-2008.aspx. Accesado en 07/11/2015. 


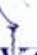

- López, M.G.; Rubio, M.S. \& Váldez, S.E. Efecto del cruzamiento, sexo y dieta en la composición química de la carne de ovinos Pelibuey con Rambouillet y Suffolk.Veterinaria México, v.31, n.1, p.11-19, 2000. Disponible en: http://www.fmvz.unam.mx/fmvz/revvetmex/a2000/rvmv31n1/rvm31102.pdf. Accesado en $10 / 07 / 2016$.

- Llorente, E.; Ramos, L. Determinación de la curva de crecimiento en ovinos criollos utilizando el modelo Brody en un sistema de producción en Córdoba. Montería, Colombia: Universidad de Córdoba, 2014. 44 p. (Trabajo de grado), Medicina Veterinaria y Zootecnia.

- Pineda, S.; Díaz, A.; Sepúlveda, J. et al. Curvas de crecimiento post-destete en el ganado Senepol de Colombia. Revista Lasallista de Investigación, v.10, n.1, p. 9-17, 2013. Disponible en: http://www.scielo.org.co/pdf/rlsi/v10n1/v10n1a02.pdf. Accesado en $10 / 07 / 2016$.

- Rocha, J.L.; Regazzi, A.J.; De Sousa, W.H. et al. Estudo da curva de crescimento de ovinos Santa Inês. Revista Brasileira de Zootecnia, v.35, n.2: 435-442, 2006. Disponible en: http://www.scielo.br/pdf/rbz/v35n2/a14v35n2.pdf. Accesado en: 10/07/2016.

- Santos, L.M. Crescimento de ovinos de diferentes grupos genéticos sob modelos não lineares convencionais e alternativos. Itapetinga, Brasil: Universidad del Suroeste de Bahía, 2012. 55p. Tese (Mestrado em Zootecnia). Disponible en: http://www.uesb.br/ppz/defesas/2012/mestrado/lorena-mirelle.pdf. Accesado en 10/07/2016.

- Statistical Analysis System Institute (SAS). SAS/STAT User's guide (Relase 9.1.3), Cary, NC, USA. 2007

- Teixeira, M.R.; Cruz, J.F.; Faria, H.H. et al. Descrição do crescimento de ovinos Santa Inês utilizando modelos não-lineares selecionados por análise multivariada. Revista Brasileira de Saúde e Produção Animal, v.17, n.1, p. 26-36. 2016.

- Vivas, N.J. Diversidad genética de ovinos criollos colombianos. Palmira, Colombia: Universidad Nacional de Colombia, 2013. 152p. Tesis (Maestría en Ciencias Agrarias). Disponible en: http://www.bdigital.unal.edu.co/12683/1/7410001.2013.pdf. Accesado en: $10 / 07 / 2016$. 
Cómo citar: Vergara-Garay, O.D.; Hincapié-Gutiérrez, L.C.; Vallejo-Romero, D.A.; Simanca-Sotelo, J.C; \& Bustamante-Yánez M.J. Utilización del modelo Brody para describir el crecimiento de dos grupos raciales de ovinos en Córdoba, Colombia. Revista Veterinaria y Zootecnia, v. 11, n. 1, p. 01-12. DOI: 10.17151/vetzo.2017.11.1.1 